\title{
Behavioral Differentiation between Anas poecilorhyncha and Domestic Duck
}

\author{
Shenglin Yang ${ }^{1}$, Lin Zhou ${ }^{1}$, Wei Lin ${ }^{1}$, Xiaomeng $\mathrm{Li}^{2}$, Man $\mathrm{Lu}^{2}$ and Chenggang $\mathrm{Liu}^{2}$ \\ 1. College of Animal Science, Guizhou University, Guiyang 550025, People's Republic of China \\ 2. Key Laboratory of Animal Genetics, Breeding and Reproduction in Plateau Mountainous Region, Ministry of Education, Guizhou \\ University, Guiyang 550025, Guizhou Province, People’s Republic of China
}

\begin{abstract}
Anas poecilorhyncha is one of improved variety of mallards, which was the protected bird species listed by International Union for Conservation of Nature (IUCN). Little is known until now about behavioral characteristics of the mallard. The objective of this study was to compare the behavioral differentials between A. poecilorhyncha and domestic duck in order to make a strategy to manage A. poecilorhyncha under the condition of animal welfare. A total of 180 birds were distributed into six groups with 30 birds for each group. They were placed in a room of $3 \mathrm{~m} \times 4 \mathrm{~m}$ and fed for one year old. Sansui ducks, a kind of native domestic duck (Sansui laying duck) were used for behavioral comparison. Two different rooms in the same building were applied, one room for $A$. poecilorhyncha and another one for Sansui ducks. All behaviors for A. poecilorhyncha and Sansui ducks were coded using the program The Observer XT 11.5 (Noldus Information Technology, Beijing). The duration of observation was from 8:00 am to 18:00 pm daily and lasted $5 \mathrm{~d}$. The results showed that there were similar behavioral percentages between A. poecilorhyncha and domestic duck. Percentages of standing activity spent for A. poecilorhyncha and Sansui duck were $34.59 \%$ and $30.25 \%$, respectively. Accordingly, the activities, such as wing plugging, preening and head stretching, were more than $5.51 \%$. The other activities, including walking, drinking and tail wagging, occupied less proportions $(\leq 3 \%)$. While the specific behaviors, like aggression, alerting, wing dithering, clawing, nodding, pendulum clawing and crawling, took less than $1 \%$ of percentage. There were large differences between A. poecilorhyncha and domestic duck when comparing eight behavioral peaks. Lag sequential analysis was used to calculate frequency of transition between a pair of activities. Some parameters were very significant, like the frequency value from foraging converted to drinking in Sansui duck was 369-515, but 37-65 in A. poecilorhyncha. The best explaining could be given that Sansui duck was better domesticated than spot-billed duck. This study provides the basic data to study and develop spot-billed duck.
\end{abstract}

Key words: Behavioral ethogram, Anas poecilorhyncha, Sansui duck, lag sequential analysis.

\section{Introduction}

Anas poecilorhyncha, a sort of mallard found in 1861-1862, is mainly distributed in the Eastern Asia, Indo-China Peninsula and India [1, 2]. There are two academic views about the origin and evolution of Chinese domestic ducks. Some scholars hold that Chinese domestic ducks originated from wild mallards (A. platyrhynchos), while others argued that Chinese domestic ducks originated from the hybrid of wild mallards (A. platyrhynchos) and spot-billed duck (A. zonorhyncha), domesticated in different areas or domesticated from hybrids of $A$. platyrhynchos and $A$.

Corresponding author: Shenglin Yang, professor, research field: animal genetics, especially in native animals. zonorhyncha [3, 4]. However, there is still no consensus conclusion about this. A few of reports have been found related to the genetic relationship between $A$. poecilorhyncha and A. platyrhynchos. Kulikova et al. [5] reported the genetic diversity and phylogenetic relationships in two groups of river ducks - A. platyrhynchos and A. poecilorhyncha using random amplified polymorphic DNA-polymerase chain reaction (RAPD-PCR), and the genetic distance between them was 0.401 , suggesting that there were a low genetic differentiation and a close evolutionary relationship between $A$. platyrhynchos and $A$. poecilorhyncha.

According to the regulation of wild animal 
conservation of China, wild animals through artificial rearing can be conducted after they reproduce into the second generation. In fact, mallard has been utilized many years because of its good tolerance to crude feed and good quality of meat, especially its plenty of essential amino acids. Some researches concerning the productive traits of $A$. poecilorhyncha have been conducted in recent years. Hu et al. [6] studied laying rhythm of A. poecilorhyncha and found that the mallard started to lay in six months old with 16 weeks of laying duration and 30 eggs approximately per year. The other productive performances, such as growth rate, reproductive rate, nutritive requirement and domesticating technique, have been investigated [7, 8]. Zhou et al. [9] investigated the whole mitochondrial genome of A. poecilorhyncha in 2015. Lavretsky et al. [10] studied the phylogenetics between the mallards and allies using molecular method in 2014. However, up to now, little is known about behavioral characteristics of the mallard. As we know, ethogram is the description to the characteristics of the behavior for some species $[11,12]$. It provides the standardized classification and definition for relevant job, so that proceeds quantitative analysis to understand the behavior, existence and breeding of animals [13, 14]. The objective was to compare the behavioral differentials between A. poecilorhyncha and domestic duck so as to make a strategy to manage $A$. poecilorhyncha under the condition of animal warfare.

\section{Materials and Methods}

\subsection{Subjects}

The experiment was performed in the Veterinary Hospital of Guizhou University, Guizhou province, China. All mallards used for this trail were bought from Poyang mallard breeding farm in Jiangxi province. A total of 180 birds were distributed into six groups with 30 birds for each group, and fed in the room of $3 \mathrm{~m} \times 4 \mathrm{~m}$ until one year old. To understand the specific behavior for the mallards, Sansui duck, a kind of native domestic duck (Sansui laying duck) was used as for behavioral comparison. Two different rooms but the same building were applied, one for $A$. poecilorhyncha and another one for Sansui ducks. To acquire all behavioral definitions, a pre-observation experiment was conducted before starting the formal observation. The formal observation experiment was conducted using 30 Sansui ducks and 30 mallards by Samsung PC camera with Media Recorder Software (Noldus Information Technology, Beijing). The duration of observation was from 8:00 am to $18: 00 \mathrm{pm}$ and lasted $5 \mathrm{~d}$. All behaviors for A. poecilorhyncha and Sansui ducks were coded using the program The Observer XT 11.5 (Noldus Information Technology, Beijing). A total of 21 sorts of behaviors for A. poecilorhyncha and 17 sorts of behaviors for Sansui ducks were coded, and each behavior could not occur simultaneously. Both instantaneous observation and continuous observation methods were applied in this study. A time unit $60 \mathrm{~s}$ was used to record the behavioral times, and 60 times $/ \mathrm{h}$ and 600 times/d were recorded, lasting $5 \mathrm{~d}$.

\subsection{Behavioral Description and Definition}

Behaviors were described and defined according to the function of the described behaviors, and data were from pre-observation and the previous studies on duck's behavior $[15,16]$. A total of 21 sorts of behaviors including A. poecilorhyncha and Sansui duck were defined, and listed in Table 1.

\subsection{Statistical Analysis}

To check reliability of data, it was necessary to run the same data more than two times or to analyze data by more than two people. It was regarded as available (Nuldus) while the reliability value was more than $75 \%$. In this experiment, the reliability values were $99.87 \%, \quad 99.81 \%, \quad 99.85 \%, \quad 99.82 \%, \quad 99.85 \%$, respectively, so these data were available for subsequent work. Behavioral data were used as statistical and correlative analysis with The Observer XT 11.5 and SPSS 18.0. The total behavioral frequency 
Table 1 Ethogram of behaviors of A. poecilorhyncha and Sansui duck.

\begin{tabular}{ll}
\hline Behavior & Definition \\
\hline Walking & Walking without performing other behavior \\
Standing & A bird is standing on the ground with two feet, maintaining an erect upright position \\
Lying & A bird is lying on the floor; its belly is resting on the floor with its wings and legs tucked underneath it; \\
Head wiggling & Movement of head towards different directions \\
Foraging & A bird is pecking at food at the feeder, including brief inter-pecking intervals \\
Preening & Rubbing the beak or lower limbs over or between the feathers \\
Head stretching & A duck is stretching its head for a period of time \\
Wing plugging & A duck's beak and head are stretching under the wings \\
$\begin{array}{l}\text { Drinking } \\
\text { Paddling }\end{array}$ & A duck drinks by suction of water through the beak \\
Crawling (only & Swiping duck's web or the whole body into water, stealth a distance to emerge from the water \\
$\begin{array}{l}\text { A. poecilorhyncha) } \\
\text { Wing dithering }\end{array}$ & A bird's body is moving in a slowly velocity when it is lying on the ground; its legs are curled underneath wings \\
Aggression & A duck is jittering its wings ceaselessly \\
Tail wagging & Stretching out by head and pecking other individuals \\
Alerting (only & A duck is moving his tail from left side to right side \\
$\begin{array}{l}\text { A. poecilorhyncha) } \\
\text { Clawing }\end{array}$ & A quiet upright waking posture with the animal standing on both legs, with both eyes showing fast blinks, \\
befecating & A bird is tickling the duck's head with palm \\
Nodding & Releasing feces from cloaca \\
Pendulum clawing & A duck is moving its head slightly downwards \\
Running & A duck is wiggling its a palm when standing with one leg \\
Flying & The action or movement of duck by foot at fast velocity \\
\hline
\end{tabular}

for A. poecilorhyncha and Sansui duck was 3,000 times. The other data analysis concerning behavioral percentage, time, frequency and lag sequence was calculated by The Observer XT program.

\section{Results}

\subsection{Behavioral Percentage and Change Occurred}

The proportions of all behaviors spent both for $A$. poecilorhyncha and Sansui duck were listed in Table 2. Percentages of standing activity spent for $A$. poecilorhyncha and Sansui duck are $30.25 \%$ and $34.59 \%$, respectively. Lying took up the second large percentage, $27.02 \%$ and $20.75 \%$, respectively. Accordingly, the activities, such as wing plugging, preening, and head stretching, were more than 5.51\%. The other activities, including walking, drinking and tail wagging, occupied less proportions $(\leq 3 \%)$. While the specific behaviors, like aggression, alerting, wing dithering, clawing, nodding, pendulum clawing and crawling, took less than $1 \%$ of percentage. Particularly, a few of frequencies for pendulum clawing and paddling could be observed for A. poecilorhyncha (spot-billed duck).

Frequencies of all behaviors occurred each day and total frequencies for $5 \mathrm{~d}$ were displayed in Fig. 1, which recorded the activities occurred for both of $A$. poecilorhyncha and Sansui ducks from day 1 to day 5 at day time. The main behaviors spent each day were largely similar. Fig. 2 represented the principal behavioral change both of A. poecilorhyncha and Sansui ducks from day 1 to day 5 . The frequency of mainly behavior-standing spent for $A$. poecilorhyncha was relatively stable (Fig. $2 \mathrm{~b}$ ), and the similar situations could be detected in smaller percentage of behavior occurred, like walking and preening (Figs. 2a and 2f). Unstable behavioral changes could be found in lying, heading dithering, foraging, drinking and wing plugging. However, it needs the further confirmation by doing a longer duration experiment. 
Table 2 Proportions of behaviors spent from day 1 to day 5 for A. poecilorhyncha and Sansui duck.

\begin{tabular}{|c|c|c|c|c|c|}
\hline \multirow{2}{*}{ Behavior } & \multicolumn{2}{|c|}{ Sansui duck } & \multicolumn{2}{|c|}{ A. poecilorhyncha } & \multirow{2}{*}{$P$ value } \\
\hline & Frequency & Proportion (\%) & Frequency & Proportion (\%) & \\
\hline Standing & 5,428 & 34.59 & 4,523 & 30.25 & 0.058 \\
\hline Lying & 3,257 & 20.75 & 4,040 & 27.02 & 0.068 \\
\hline Wing plugging & 1,929 & 12.3 & 2,217 & 14.83 & 0.044 \\
\hline Head wiggling & 715 & 4.56 & 1,099 & 7.35 & 0.133 \\
\hline Preening & 959 & 6.11 & 1,076 & 7.20 & 0.037 \\
\hline Head stretching & 1,183 & 7.54 & 824 & 5.51 & 0.113 \\
\hline Foraging & 980 & 6.24 & 418 & 2.80 & 0.243 \\
\hline Walking & 387 & 2.47 & 285 & 1.91 & 0.096 \\
\hline Drinking & 439 & 2.80 & 152 & 1.02 & 0.288 \\
\hline Tail wagging & 265 & 1.69 & 133 & 0.89 & 0.204 \\
\hline Aggression & 11 & 0.07 & 78 & 0.52 & 0.411 \\
\hline Clawing & 37 & 0.24 & 34 & 0.23 & 0.027 \\
\hline Alerting & 14 & 0.08 & 33 & 0.22 & 0.245 \\
\hline Wing dithering & 56 & 0.36 & 22 & 0.15 & 0.262 \\
\hline Defecating & 9 & 0.05 & 8 & 0.05 & 0.037 \\
\hline Nodding & 8 & 0.05 & 4 & 0.03 & 0.205 \\
\hline Crawling & 1 & 0.01 & 0 & 0.02 & 0.500 \\
\hline Pendulum clawing & 8 & 0.05 & 1 & 0.00 & 0.421 \\
\hline Paddling & 7 & 0.04 & 3 & 0.00 & 0.242 \\
\hline Total & 15,693 & 100.00 & 14,950 & 100.00 & 0.015 \\
\hline
\end{tabular}

3.2 Lag Sequential Analysis of A. poecilorhyncha and Sansui Duck

In The Observer XT 11.5, lag sequential analysis calculates the frequency of transitions between pairs of events within a certain lag. The first event of the pair is called criterion and the second is target. Depending on what direction in time you choose (positive or negative), we can calculate how often the event $\mathrm{A}$ is followed by $\mathrm{B}$, or how often $\mathrm{A}$ is preceded by $\mathrm{B}$, and in relation to other behaviors. Here, the frequency was selected instead of probability; in the result of matrix, each row represents a criterion activity and each column is a target activity (Fig. 3).

From Table 3, it can be seen that the frequency for standing for A. poecilorhyncha with two legs transited to walking activity was 1,710 and walking converted to standing with two legs occurred 1,724 times. While, the frequency of walking to standing with two legs in Sansui duck was 3,758 and from standing to walking was 3,715 (Table 4). It can be seen that transition for standing and walking activity was easy to be detected both in A. poecilorhyncha and Sansui duck, but more often in Sansui duck than that in A. poecilorhyncha. Similarly, frequency for standing with two legs converted to standing with one leg was 536 and from standing with one leg to standing with two legs was 512 times for A. poecilorhyncha (Table 3). But frequency for standing transited to running or flying were zero, while walking to running was two, no standing process from walking to running or flying. Conversion from lying to walking was 49 and from walking to lying was 36 ; it can be explained that transition between walking and lying was often and easy. In Sansui duck, from standing with two legs to running was three and no flying activity could be observed (Table 4). But, the frequency of transition from lying to standing was often to be observed both in two sorts of ducks (296 and 318, respectively). Interestingly, the frequencies from standing to running both for A. poecilorhyncha and Sansui duck were zero, except standing with two legs to running in Sansui 
274

Behavioral Differentiation between Anas poecilorhyncha and Domestic Duck

a

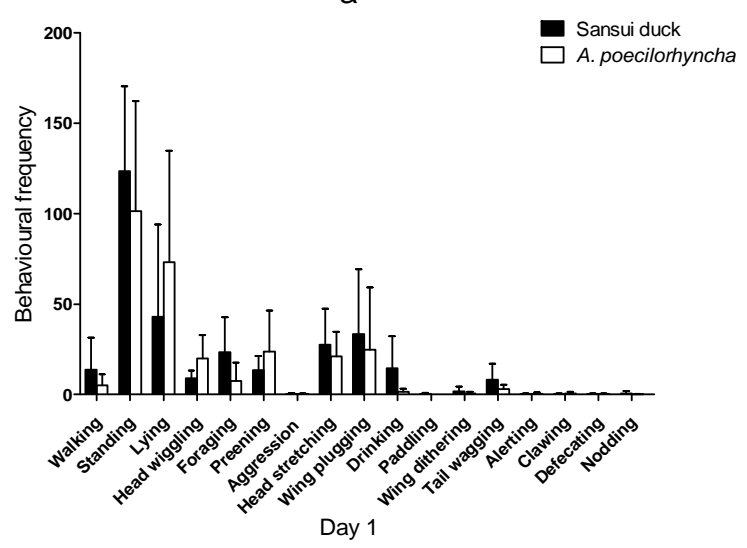

C

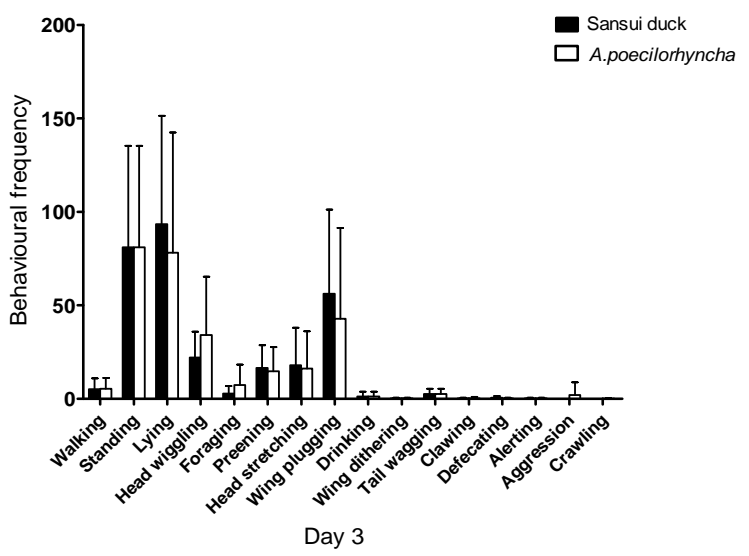

e

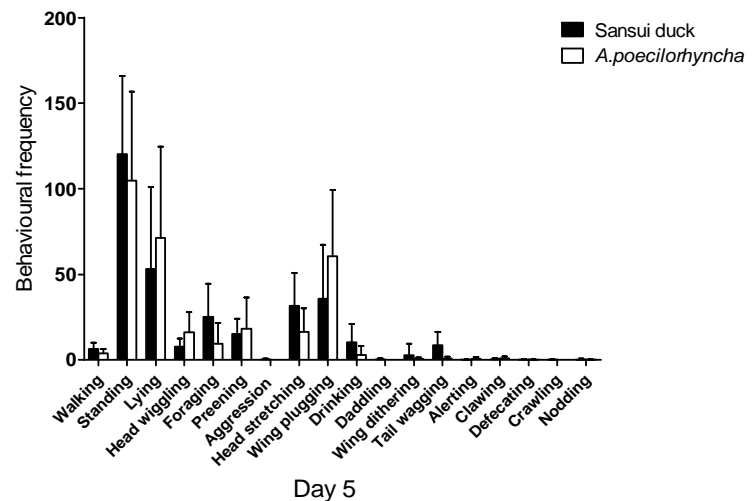

b

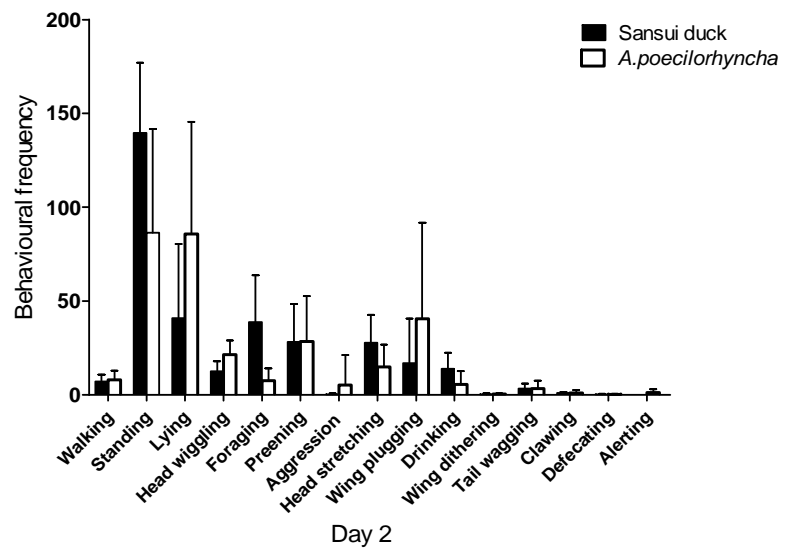

Day 2

d

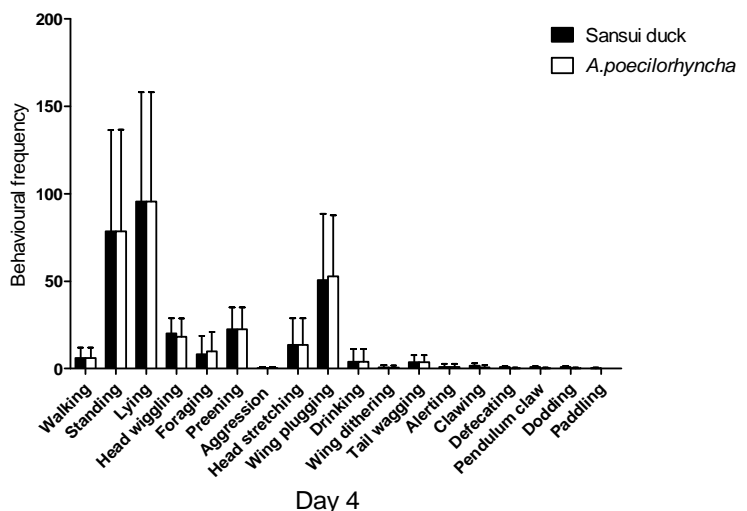

Day 4

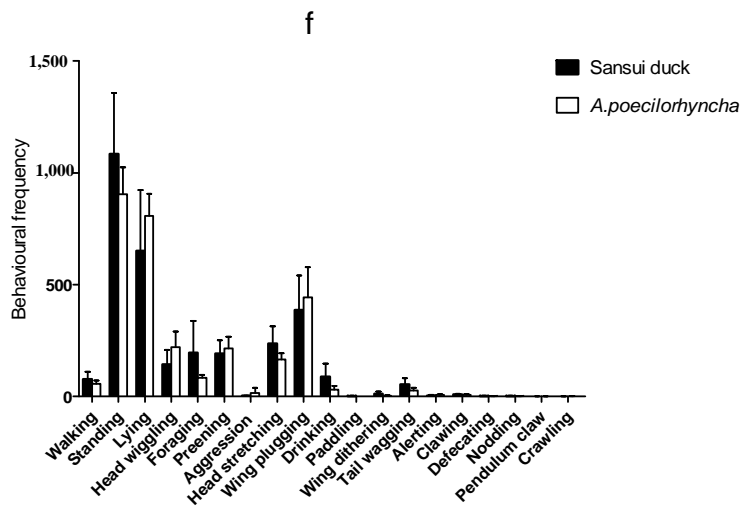

Day 1-5

Fig. 1 Behavioral frequency of observation from day 1 to day 5 (are) and total behavioural frequency of $5 \mathrm{~d}$ (f) for $\mathrm{A}$. poecilorhyncha and Sansui duck in the experiment. 
a

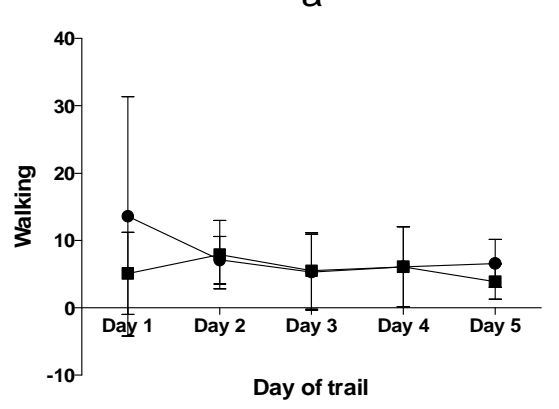

C

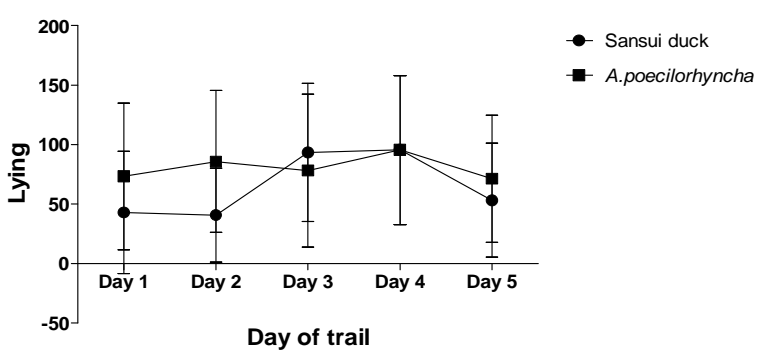

e

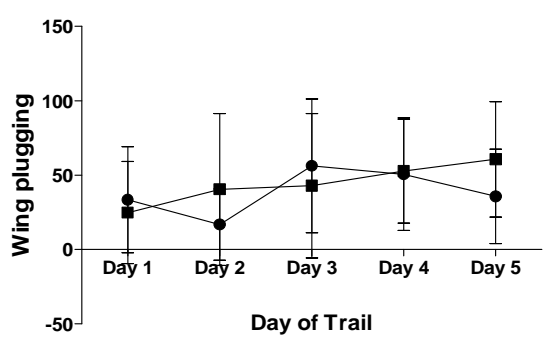

- Sansui duck

- A.poecilorhyncha b

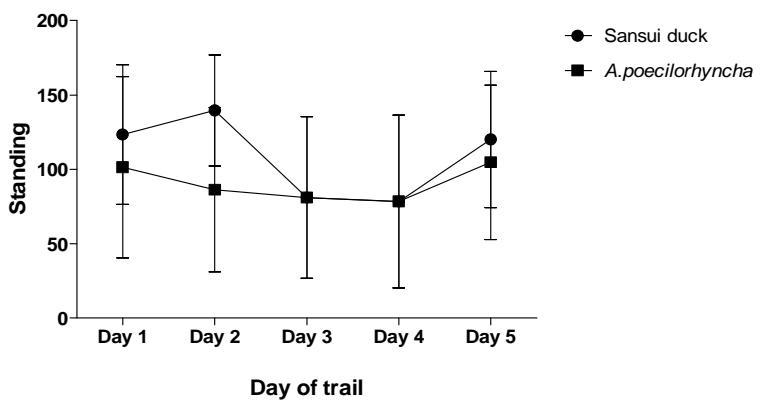

d

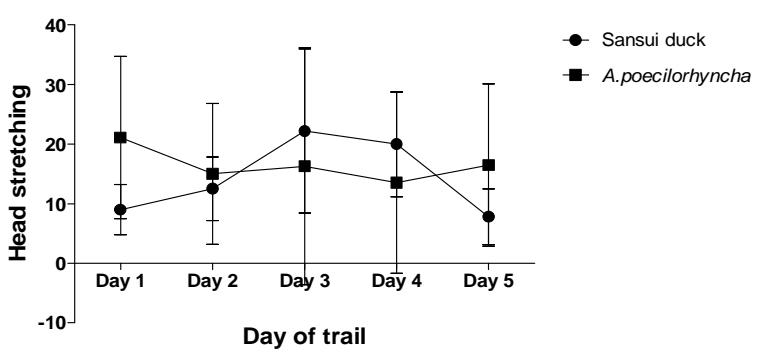

$\mathbf{f}$

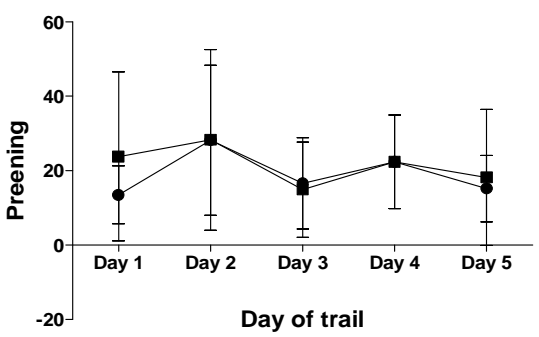

h

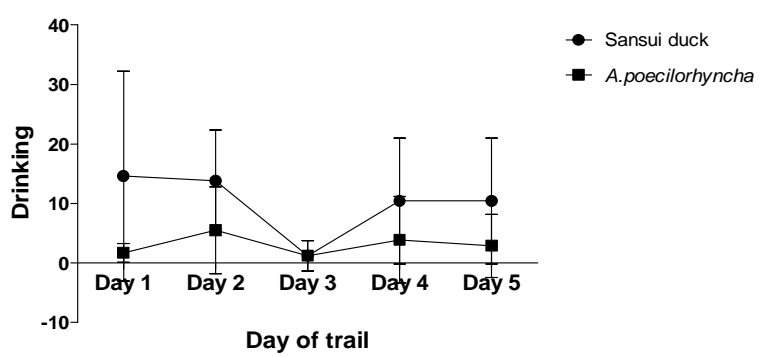

Fig. 2 Principal behavioral change of proportions spent from day 1 to day 5 for A. poecilorhyncha and Sansui duck. 


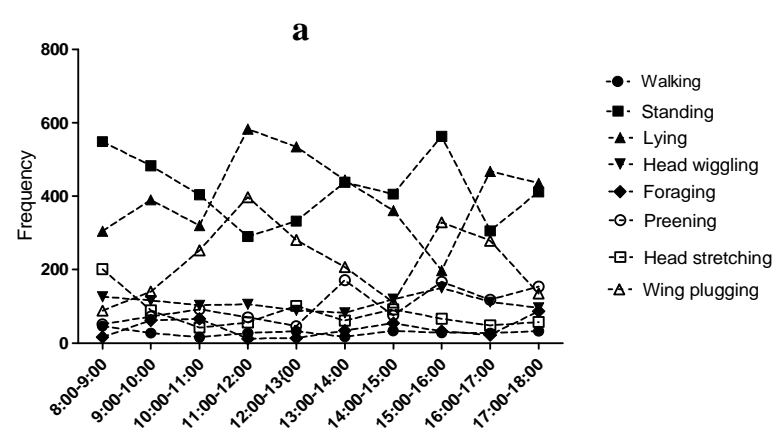

Time (8:00 am to $18: 00 \mathrm{pm})$

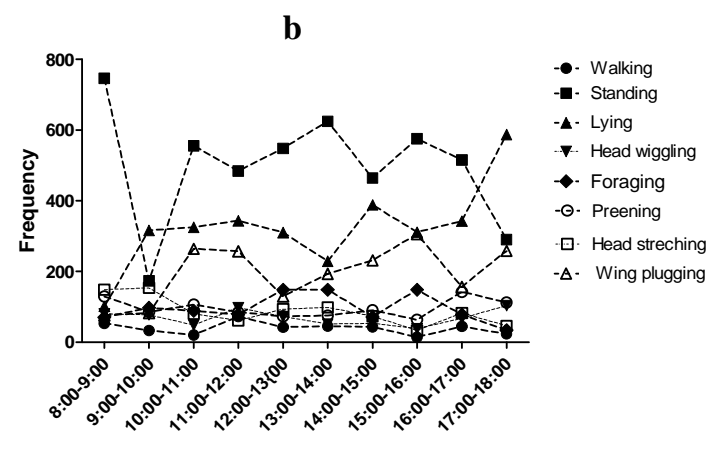

Time (8:00 am to $18: 00 \mathrm{pm})$

Fig. 3 The diurnal activities frequency of main behavior both in A. poecilorhyncha (a) and Sansui duck (b).

duck was three, suggesting that running activity does not require standing as a preparatory activity.

From Tables 5 and 6, the frequency for head wiggling converted to preening was 273 in spot-billed duck, but 132 in Sansui duck. The frequency of head wiggling transited to head stretching in spot-billed was 266, but 150 in Sansui duck. Frequency conversion from foraging to head stretching was 127 in spot-billed duck, but 472 in domestic duck. This may be explained that domestic duck has more comfortable time than wild duck after foraging. Transition from forging to dinking for spot-billed dick was 65 , but 515 in domestic duck, which means that domestic duck needs much more time to drink than wild duck after foraging. It may be concluded that wild duck has stronger ability to adapt natural environment than domestic duck.

The transition values can be compared for small percentage of behavior. The transition frequency between foraging and head wiggling was 63 to 69 in spot-billed duck, but 167 to 217 in Sansui duck. The transition frequency between preening to head stretching was 66 to 91, while 94 to 95 in Sansui duck. Drinking to head stretching was 75 to 82 , but 240 to 247 in Sansui duck. Drinking to head wiggling was 65 to 86 , but 125 to 143 in Sansui duck. The larger differences of frequencies transition could be observed between spot-billed duck and Sansui duck. The other transitions concerning nodding and clawing, aggression and clawing, foraging and nodding, etc., could not be found both in spot-billed duck and Sansui duck. About some specific behaviors, such as paddling and aggression, a few of transitions occurred due to their smaller percentage of activities in this study.

\subsection{Correlations of Behavioral Activities of A. poecilorhyncha}

The Pearson correlation analysis in $A$. poecilorhyncha was listed in Table 7 . It showed that drinking and alerting, aggression and clawing, paddling and aggression, aggression and wing dithering, paddling and wing dithering were significant correlative $(R=0.981, P<0.01 ; R=0.917$, $P<0.01 ; R=0.913, P<0.05 ; R=0.942, P<0.05 ; R$ $=0.975, P<0.01)$. Conversely, lying and standing, nodding and lying, tail wagging and nodding were significant negative correlation $(R=-0.996, P<0.01$; $R=-0.895, P<0.01 ; R=-0.925, P<0.01)$.

\subsection{The Diurnal Activities of A. poecilorhyncha and Sansui Duck}

Fig. 3 showed that the eight main behaviors of $A$. poecilorhyncha were included from the time 8:00 am to $18: 00 \mathrm{pm}$, and the highest frequencies for standing achieved 563 at 15:00 to $16: 00 \mathrm{pm}$, while the lowest was 290 at 11:00 to 12:00 am. However, the standing peak for Sansui duck at 8:00 to 9:00 am with a frequency value of 746, and the lowest value was 173 at 9:00 to 10:00 am. The activity lying peak for wild duck was at 11:00 to 12:00 am with the total frequency 583 , and the lowest frequency was 198 at 15:00 to 
Table 3 Lag sequential analysis of A. poecilorhyncha (group I).

\begin{tabular}{|c|c|c|c|c|c|c|c|c|}
\hline Behavior & Walking & Running & Flying & Circling & Standing with two legs & Standing with one leg & Lying & $Y_{0}$ \\
\hline Walking & 29 & 2 & 2 & 0 & 1,724 & 64 & 36 & 1 \\
\hline Running & 3 & 0 & 0 & 0 & 0 & 0 & 0 & 0 \\
\hline Flying & 0 & 0 & 0 & 0 & 2 & 0 & 0 & 0 \\
\hline Circling & 0 & 0 & 0 & 0 & 1 & 0 & 1 & 0 \\
\hline Standing with two legs & 1,710 & 0 & 0 & 0 & 2,599 & 536 & 292 & 12 \\
\hline Standing with one leg & 65 & 0 & 0 & 2 & 512 & 605 & 241 & 2 \\
\hline Lying & 49 & 0 & 0 & 0 & 296 & 220 & 3,669 & 5 \\
\hline $\begin{array}{l}X_{0} \\
\end{array}$ & 0 & 0 & 0 & 0 & 15 & 0 & 0 & 0 \\
\hline
\end{tabular}

$X_{0}$ refers to transitions where a target activity has been identified, but the criterion activity is not found. $Y_{0}$ refers to transitions where no target activity is found.

Table 4 Lag sequential analysis for Sansui duck (group I).

\begin{tabular}{|c|c|c|c|c|c|c|}
\hline Behavior & Walking & Running & Standing with two legs & Standing with one leg & Lying & $Y_{0}$ \\
\hline Walking & 16 & 8 & 3,758 & 35 & 61 & 0 \\
\hline Running & 14 & 0 & 0 & 0 & 0 & 0 \\
\hline Standing with two legs & 3,715 & 3 & 5,147 & 434 & 317 & 18 \\
\hline Standing with one leg & 41 & 0 & 399 & 232 & 93 & 0 \\
\hline Lying & 92 & 3 & 318 & 64 & 1,910 & 2 \\
\hline$X_{0}$ & 0 & 0 & 12 & 0 & 8 & 0 \\
\hline
\end{tabular}

$X_{0}$ refers to transitions where a target activity has been identified but the criterion is not found. $Y_{0}$ refers to transitions where no target activity is found. 
Table 5 Lag sequential analysis of A. poecilorhyncha (group II).

\begin{tabular}{|c|c|c|c|c|c|c|c|c|c|c|c|c|c|}
\hline Behavior & $\begin{array}{l}\text { Head } \\
\text { wiggling }\end{array}$ & Forging & Preening & Alerting & Clawing & Aggression & Nodding & $\begin{array}{l}\text { Head } \\
\text { dithering }\end{array}$ & $\begin{array}{l}\text { Head } \\
\text { stretching }\end{array}$ & $\begin{array}{l}\text { Wing } \\
\text { plugging }\end{array}$ & Drinking & Paddling & $Y_{0}$ \\
\hline Head wiggling & 216 & 63 & 273 & 6 & 20 & 6 & 0 & 3 & 266 & 115 & 65 & 0 & 2 \\
\hline Forging & 69 & 97 & 26 & 1 & 10 & 6 & 1 & 0 & 127 & 5 & 65 & 0 & 0 \\
\hline Preening & 254 & 27 & 171 & 0 & 28 & 0 & 1 & 1 & 91 & 29 & 42 & 0 & 4 \\
\hline Alerting & 7 & 2 & 2 & 3 & 0 & 0 & 0 & 0 & 12 & 0 & 0 & 0 & 0 \\
\hline Clawing & 34 & 10 & 23 & 0 & 4 & 0 & 0 & 7 & 19 & 4 & 12 & 0 & 0 \\
\hline Aggression & 10 & 3 & 0 & 1 & 0 & 0 & 0 & 0 & 7 & 0 & 3 & 0 & 0 \\
\hline Nodding & 3 & 0 & 0 & 0 & 0 & 0 & 0 & 0 & 6 & 2 & 0 & 0 & 0 \\
\hline Head dithering & 3 & 0 & 1 & 0 & 1 & 0 & 0 & 0 & 49 & 0 & 0 & 0 & 0 \\
\hline Head stretching & 267 & 139 & 66 & 14 & 29 & 10 & 6 & 41 & 315 & 8 & 76 & 1 & 5 \\
\hline Wing plugging & 142 & 7 & 38 & 0 & 4 & 0 & 2 & 0 & 19 & 1,763 & 3 & 0 & 3 \\
\hline Drinking & 86 & 37 & 33 & 2 & 25 & 4 & 0 & 0 & 82 & 0 & 23 & 0 & 1 \\
\hline Paddling & 0 & 0 & 0 & 0 & 0 & 0 & 0 & 1 & 2 & 0 & 1 & 0 & 0 \\
\hline$X_{0}$ & 1 & 0 & 1 & 0 & 0 & 0 & 0 & 0 & 0 & 0 & 0 & 0 & 0 \\
\hline
\end{tabular}

$X_{0}$ refers to transitions where a target activity has been identified but the criterion is not found; $Y_{0}$ refers to transitions where no target activity is found.

Table 6 Lag sequential analysis of Sansui duck (group II).

\begin{tabular}{|c|c|c|c|c|c|c|c|c|c|c|c|c|}
\hline Behavior & $\begin{array}{l}\text { Head } \\
\text { wiggling }\end{array}$ & Forging & Preening & Clawing & Aggression & Nodding & $\begin{array}{l}\text { Head } \\
\text { dithering }\end{array}$ & $\begin{array}{l}\text { Head } \\
\text { stretching }\end{array}$ & $\begin{array}{l}\text { Wing } \\
\text { Plugging }\end{array}$ & Drinking & Paddling & $Y_{0}$ \\
\hline Head wiggling & 55 & 167 & 132 & 3 & 0 & 5 & 1 & 150 & 42 & 125 & 6 & 0 \\
\hline Forging & 217 & 614 & 61 & 17 & 8 & 0 & 0 & 472 & 45 & 515 & 1 & 5 \\
\hline Preening & 112 & 77 & 131 & 36 & 0 & 1 & 0 & 95 & 33 & 23 & 0 & 2 \\
\hline Clawing & 26 & 31 & 3 & 0 & 0 & 0 & 10 & 12 & 0 & 29 & 0 & 0 \\
\hline Aggression & 0 & 5 & 0 & 0 & 0 & 0 & 0 & 3 & 1 & 1 & 0 & 0 \\
\hline Nodding & 0 & 0 & 0 & 0 & 0 & 2 & 0 & 2 & 2 & 4 & 0 & 0 \\
\hline Head dithering & 6 & 3 & 13 & 0 & 0 & 0 & 0 & 19 & 0 & 8 & 3 & 0 \\
\hline Head Stretching & 151 & 520 & 94 & 11 & 0 & 0 & 20 & 547 & 29 & 240 & 7 & 2 \\
\hline Wing plugging & 41 & 52 & 43 & 0 & 0 & 0 & 0 & 49 & 1,020 & 21 & 0 & 0 \\
\hline Drinking & 143 & 396 & 35 & 27 & 5 & 1 & 6 & 247 & 23 & 133 & 40 & 2 \\
\hline Paddling & 7 & 2 & 0 & 0 & 0 & 0 & 0 & 27 & 3 & 33 & 0 & 0 \\
\hline$X_{0}$ & 0 & 0 & 0 & 0 & 0 & 0 & 0 & 17 & 0 & 1 & 0 & 0 \\
\hline
\end{tabular}

$X_{0}$ refers to transitions where a target activity has been identified but the criterion is not found; $Y_{0}$ refers to transitions where no target activity is found. 
Table 7 Correlations for frequencies of behavioral activities of $A$. poecilorhyncha.

\begin{tabular}{|c|c|c|c|c|c|c|c|c|c|c|c|c|c|c|c|c|c|}
\hline Behavior & Walking & Standing & Lying & H. Wig & Foraging & Preening & Alerting & Clawing & Aggression & Nodding & H. str & W. pl & Drinking & Padding & W. dit & T. weg & P. claw Defec \\
\hline Walking & 1 & & & & & & & & & & & & & & & & \\
\hline Standing & -0.634 & 1 & & & & & & & & & & & & & & & \\
\hline Lying & 0.566 & $-0.996^{* *}$ & 1 & & & & & & & & & & & & & & \\
\hline H. wig & 0.679 & -0.603 & 0.573 & 1 & & & & & & & & & & & & & \\
\hline Foraging & -0.123 & 0.651 & -0.687 & -0.537 & 1 & & & & & & & & & & & & \\
\hline Preening & 0.780 & -0.079 & -0.004 & 0.375 & 0.486 & 1 & & & & & & & & & & & \\
\hline Alerting & 0.707 & -0.182 & 0.112 & -0.002 & 0.517 & 0.832 & 1 & & & & & & & & & & \\
\hline Clawing & 0.128 & -0.167 & 0.157 & 0.004 & 0.465 & 0.356 & 0.346 & 1 & & & & & & & & & \\
\hline Aggression & 0.415 & -0.495 & 0.475 & 0.140 & 0.264 & 0.432 & 0.532 & $0.917^{*}$ & 1 & & & & & & & & \\
\hline Nodding & -0.806 & $0.921^{*}$ & $-0.895^{* *}$ & -0.846 & 0.573 & -0.347 & -0.24 & -0.17 & -0.456 & 1 & & & & & & & \\
\hline H. str & -0.297 & 0.466 & -0.457 & 0.331 & -0.124 & -0.122 & -0.62 & -0.33 & -0.593 & 0.209 & 1 & & & & & & \\
\hline W. pl & -0.351 & -0.200 & 0.252 & -0.499 & -0.408 & -0.708 & -0.23 & -0.413 & -0.243 & 0.161 & -0.50 & 1 & & & & & \\
\hline Drinking & 0.710 & -0.238 & 0.171 & -0.031 & 0.404 & 0.746 & $0.981^{* *}$ & 0.197 & 0.436 & -0.262 & -0.684 & -0.066 & 1 & & & & \\
\hline Padding & 0.182 & -0.565 & 0.576 & -0.053 & 0.066 & 0.060 & 0.321 & 0.799 & $0.913^{*}$ & -0.375 & -0.728 & 0.145 & 0.278 & 1 & & & \\
\hline T. weg & 0.856 & -0.776 & 0.732 & 0.804 & -0.238 & 0.607 & 0.447 & 0.456 & 0.666 & $-0.925^{*}$ & -0.211 & -0.446 & 0.394 & 0.469 & 0.422 & 1 & \\
\hline P. claw & -0.043 & -0.035 & 0.038 & 0.110 & 0.377 & 0.224 & 0.048 & $0.932^{*}$ & 0.745 & -0.102 & 0.012 & -0.552 & -0.127 & 0.612 & 0.656 & 0.376 & 1 \\
\hline Defec & -0.260 & 0.070 & -0.040 & 0.521 & -0.465 & -0.322 & -0.786 & -0.186 & -0.373 & -0.102 & 0.875 & -0.356 & -0.838 & -0.408 & -0.515 & 0.021 & 0.167 \\
\hline
\end{tabular}

H. wig = heading wiggling; H. str = head stretching; W. pl = wing plugging; W. dit = wing dithering; T. weg = tail wegging; P. claw = pendulum clawing; Defec: defecating. 
16:00 pm. While, the lying peak for Sansui duck was at $17: 00$ to $18: 00 \mathrm{pm}$ with the value of 587 , and the lowest value was 101 at 8:00 to 9:00 am. The walking peak for wild duck at 16:00 to 17:00 pm with frequency value of 118 , and the lowest value was 27 at 9:00 to 10:00 am. In Sansui duck, the walking peak at 11:00 to $12: 00$ am with the value of 72 , while the lowest value was 14 at 15:00 to 16:00 pm. There were larger differences between A. poecilorhyncha and Sansui duck. The wing plugging for wild duck peak at 11:00 to $12: 00$ am with the value of 398 , and the lowest value was 88 at 8:00 to 9:00 am, but the largest 305 in Sansui duck at $15: 00$ to $16: 00 \mathrm{pm}$ and the smallest value was 76 at 8:00 to 9:00 am. The foraging for wild duck peak at 17:00 to 18:00 pm with the frequency value of 87 and the smallest value was 16 at 8:00 to 9:00 am. However, there were three peaks of foraging for Sansui duck at 12:00 am to 13:00 pm, 13:00 to $14: 00 \mathrm{pm}$ and $15: 00$ to $16: 00 \mathrm{pm}$ with the value of 148 . The comfortable behavior preening peak for wild duck was at 13:00 to 14:00 pm with the value of 171 and the lowest point was 46 at 12:00 am to $13: 00 \mathrm{pm}$; while 142 at 16:00 to $17: 00 \mathrm{pm}$ for Sansui duck and the lowest 63 at 15:00 to 16:00 $\mathrm{pm}$. In total, there were large differences between spot-billed duck and domestic duck when comparing the eight behavioral peaks (Fig. 3).

\section{Discussion}

4.1 Behavioral Percentages, Changes and Peaks for A. poecilorhyncha and Sansui Duck

Behavior is the well expression of animals to the environment change [17], and to understand animal ethogram can help people to know evolution history between two kinds of animals. It is necessary for people to understand animal behaviors, because it can provide ideas to make effective methods to protect and utilize them [18]. In the current study, the principal behavioral types spent between spot-billed duck and Sansui duck were similar, but percentages were not identical. A total of 21 types of behaviors for $A$. poecilorhyncha and 19 sorts of behaviors were coded and defined using the continuous and instantaneous observation methods [19-21]. The principal behavioral change both of A. poecilorhyncha and Sansui ducks was determined from day 1 to day 5 in this study. The frequency of mainly behavior, standing spent for $A$. poecilorhyncha was stable (Fig. 2b), the other behaviors were relatively unstable. There are a large number of reports related to genetic structure and growth performance of mallards comparing with other domestic ducks [22, 23]. However, a few of reports concerning the behavior of mallards could be found. It is difficult to compare with the other papers which refer to behavior of $A$. poecilorhyncha, although the report in this study is primary.

The behavioral peaks spent among eight main behaviors both for A. poecilorhyncha and Sansui ducks were compared. Results showed the differences between spot-billed duck and Sansui laying duck. Some rules can be found by use of study on behavioral rhythm, for instance, the preening peak at 13:00 to $14: 00 \mathrm{pm}$ and $15: 00$ to $16: 00 \mathrm{pm}$ for $A$. poecilorhyncha, and the forging peak at 12:00 am to 13:00 pm both for A. poecilorhyncha and Sansui duck. This can be explained by the fact that they have two different origins and different genetic structures [18, 22]. Additionally, behavior is the complicated trait which is controlled by genes. So, animals displaying their behavioral performance at different time duration can be understood.

\subsection{Behavioral Transition Frequencies through Lag} Sequential Analysis

Lag sequential analysis has been widely used for studies in psychology, neurology, business and education [24-26]. Although papers concerning human behaviors in medical science or management behaviors in business can be found in many sorts of journals, up to now, no reports in relevant to animal behavior using lay sequential analysis could be found. In the present study, lag sequential analysis was used 
to calculate frequency of transition between a pair of activities calling criterion and target (Fig. 3). Some parameters were very significant, like the frequency value from foraging converted to drinking in Sansui duck was 369-515, but 37-65 in spot-billed duck. The best explaining could be that Sansui duck was better domesticated than spot-billed duck. Domestic duck need much time to drink after foraging, but mallard could adapt to natural environment better than domestic duck. Another parameter, 36-49 from walking to lying in spot-billed duck, but 61-92 in Sansui duck, suggested that domestic duck had more percentages of comfortable behaviors than that of mallard, particularly, when they were caged in the environment of artificial rearing. This study provided an idea to study animal behavioral conversion using lag sequential analysis and to annotate how often the target behavior occurs or to predict what activity will happen.

In the current study, behaviors of spot-billed duck and Sansui duck were tracked with Media Record and The Observer XT 11.5 in captivity conditions. Compared with the method of observation by eyes, The Observer XT is more scientific and reliability. It may avoid the impact of human disturbance to investigate behavioral rule, time distribution and their correlation, to compare with the data from domestic ducks and to find the specific behavior and give the definition. This research is also the beginning of conducting the research of behavior ecology in spot-billed duck. This study provides the basic data to study and develop spot-billed duck.

\section{Conclusions}

This research has showed that there were similar behavioral percentages between A. poecilorhyncha and domestic duck through behavioural comparison. However, large differences between $A$. poecilorhyncha and domestic duck could be detected when comparing the eight behavioral peaks. An available explaining could be that Sansui duck was better domesticated than spot-billed duck.

\section{Acknowledgments}

This work was supported by the Natural Science Foundation of China (31360566), the Agricultural Major Special Project of Guizhou province (No. 6004, 2012) and the Research Project from Educational Bureau of Guizhou province (Qiankehe NY 2013, No. 120).

\section{References}

[1] Chen, Y. X., Zhao, Y., Lu, L. J., and Lai, Y. Z. 1999. "Comparative Study of Mitochondrial DNA Restriction Maps of Genus Anas Ducks in Fujian Province." $J$. Xiamen Univ. (Natural Science) 38 (1): 108-11. (in Chinese)

[2] Chen, Y. X., and Zuo, Z. H. 2001. "Study on Genetic Relationship and Diversity in Ducks by RAPD." $J$. Xiamen Univ. (Natural Science) 40 (1): 141-2. (in Chinese)

[3] Qiu, X. P. 1989. China Chicken Breeds Collection. Shanghai, China: Scientific and Technical Publishers. (in Chinese)

[4] Chang, H. 1995. Conspectus of Genetic Resources of Livestock. Beijing, China: Chinese Agriculture Press. (in Chinese)

[5] Kulikova, I. V., Chelomina, G. N., and Zhuravlev, Y. N. 2003. "Low Genetic Differentiation of and Close Evolutionary Relationships between Anas platyrhynchos and Anas poecilorhyncha: RAPD-PCR Evidence." Russian J. Genet. 39 (10): 1143-51.

[6] Hu, R. Q., Fan, Y. F., Li, Y. Q., Ma, J. S., Wang, L. M., and Cen, S. J. 2010. "Study on Laying Rhythm in Anas poecilorhyncha." Zhejiang Anim. Vet. Sci. 2: 3-4. (in Chinese)

[7] Liu, J. W., Chen, H. C., Pan, D., Deng, X. B., Zheng, S. X., and Huang, L. B. 1995. "The Comparative Anatomy of the Pancreas Lobs and Ducts of 11 Sorts of Mallards." Acta Anatomica Sinica 26 (3): 240-60. (in Chinese)

[8] Du, W. D., Li, Y. Q., Fan, Y. F., Hu, S. Q., Luo, S. Q., Seng, J. D., Lu, L. Z., and Tao, Z. R. 2006. "Study on Effects of Artificial Domesticating of Anas poecilorhyncha." Zhejiang Anim. Vet. Sci. 6: 25-6. (in Chinese)

[9] Zhou, W. L., Zhang, C. L., Pan, T., Yan, L. H., Hu, C. C., Xue, C., Chang, Q., and Zhang, B. W. 2015. "The Complete Mitochondrial Genome of Anas poecilorhyncha (Anatidae: Anas)." Mitochondrial DNA 26 (2): 265-6. 
[10] Lavretsky, P., McCracken, K. G., and Peters, J. L. 2014. "Phylogenetics of a Recent Radiation in the Mallards and Allies (Ayes: Anas): Inferences from a Genomic Transect and the Multispecies Coalescent." Mol. Phyloge. Evol. 70: 402-11.

[11] Brown, J. L. 1976. The Evolution of Behavior. New York: Norton Press.

[12] Lehner, P. N. 1996. Handbook of Ethological Methods. New York: Cambridge University Press.

[13] Martin, P., and Bateson, P. 1993. Measuring Behavior: An Introductory Guide. Cambridge: Cambridge University Press.

[14] Zhao, X. M., Ma, M., Zhang, T., and Zhang, J. B. 2013. "Behavioral Time Budget and Diurnal Rhythm of White-Headed Duck in Northwest China." Chinese Journal of Ecology 32 (9): 2439-43.

[15] Feng, S. M., and Zhang, A. J. 2000. "Behavioral Ecology of Black-Necked Crane during Winter at Caohai, Guizhou, China.” Acta Ecol. Sinica 20 (2): 293-8.

[16] Lu, X. 2004. "Anti-predation Vigilance of Individual Tibetan Eared Pheasants Temporarily Separated from the Flocks.” Acta Zoologica Sinica 50 (1): 32-6.

[17] Jiang, Z. G. 2000. "Behavior Coding and Ethogram of the Pere David's Deer." Acta Theriologica Sinica 20 (1): $1-12$.

[18] Tian, J. D., Wang, Z. L, Lu, J. Q., Guo, X. B., and Liu, J. D. 2011. "PAE Coding System-Based Ethogram of Taihangshan Macaque (Macaca mulatta tcheliensis), Jiyuan, Henan Province, China." Acta Theriologica Sinica 31 (2): 125-40.

[19] Lan, T. M., Tian, Y. P., Hu, X. N., Wang, Y. L., Jing, P., Fan, M., Zou, Q., and Liu, W. S. 2012. "Comparison between Focal-Animal Sampling and Scan Sampling for Behavioral Observation of Black Bear in Captivity." $J$. Econ. Anim. 16 (2): 95-100.

[20] Zhang, J., Chu, H. J., and Zhong, S. L. 2003. "Observation on Behavior of Mandrill (Mandrillus sphinx) in Captive." Sichuan J. Zool. 22 (2): 69-72. (in Chinese)

[21] Teng, L. W., Li, F., and Liu, Z. S. 2002. "Behavior Observation of Amur Tiger (Panthera tigris altaica) in Captivity." Journal of Forestry Research 13 (3): 241-4.

[22] Avise, J. C., Ankney, C. D., and Nelson, W. S. 1990. "Mitochondrial Gene Trees and the Evolutionary Relationship of Mallard and Black Ducks." Evolution 44 (4): 1109-19.

[23] Hou, Z. C., Yang, F. X., Qu, L. J., Zheng, J. X., Brun, J. M., Basso, B., Pitel, F., Yang, N., and Xu, G. Y. 2012. "Genetic Structure of Eurasian and North American Mallard Ducks Based on mtDNA Data." Animal Genetics 43 (3): 352-5.

[24] Ianiro, P. M., Lehmann-Willenbrock, N., and Kauffeld, S. 2015. "Coaches and Clients in Action: A Sequential Analysis of Interpersonal Coach and Client Behavior." J. Bus. Psychol. 30 (3): 435-56.

[25] Rick, D., Warlaumont, A., and Richardson, D. 2011. "Nominal Cross Recurrence as a Generalized Lag Sequential Analysis for Behavioral Streams." International Journal of Bifurcation and Chaos 21 (4): 1153-61.

[26] Hou, H. T., Chang, K. E., and Sung, Y. T. 2010. "Applying Lag Sequential Analysis to Detect Visual Behavioural Patterns of Online Learning Activities." British Journal of Educational Technology 41 (2): 25-7. 